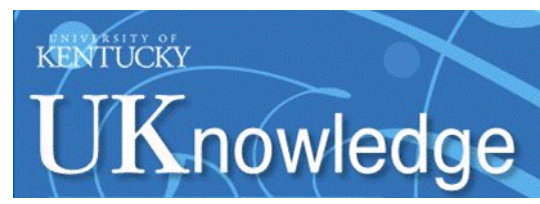

University of Kentucky

UKnowledge

7-9-2018

\title{
Laser-Induced Fluorescence Detection of the Elusive SiCF Free Radical
}

\author{
Gretchen Rothschopf \\ Ideal Vacuum Products, LLC \\ Tony C. Smith \\ Ideal Vacuum Products, LLC \\ Dennis J. Clouthier \\ University of Kentucky, dclaser@uky.edu
}

Follow this and additional works at: https://uknowledge.uky.edu/chemistry_facpub

Part of the Chemistry Commons, and the Physics Commons

Right click to open a feedback form in a new tab to let us know how this document benefits you.

\section{Repository Citation}

Rothschopf, Gretchen; Smith, Tony C.; and Clouthier, Dennis J., "Laser-Induced Fluorescence Detection of the Elusive SiCF Free Radical" (2018). Chemistry Faculty Publications. 130.

https://uknowledge.uky.edu/chemistry_facpub/130

This Article is brought to you for free and open access by the Chemistry at UKnowledge. It has been accepted for inclusion in Chemistry Faculty Publications by an authorized administrator of UKnowledge. For more information, please contact UKnowledge@lsv.uky.edu. 


\section{Laser-Induced Fluorescence Detection of the Elusive SiCF Free Radical}

\section{Digital Object Identifier (DOI)}

https://doi.org/10.1063/1.5040473

\section{Notes/Citation Information}

Published in The Journal of Chemical Physics, v. 149, issue 2, 024301, p. 1-9.

(C) 2018 Author(s)

This article may be downloaded for personal use only. Any other use requires prior permission of the author and AIP Publishing.

This article appeared in The Journal of Chemical Physics, v. 149, issue 2, 024301, p. 1-9 and may be found at https://doi.org/10.1063/1.5040473. 


\section{Laser-induced fluorescence detection of the elusive SiCF free radical}

Cite as: J. Chem. Phys. 149, 024301 (2018); https://doi.org/10.1063/1.5040473

Submitted: 17 May 2018 . Accepted: 19 June 2018 . Published Online: 09 July 2018

Gretchen Rothschopf, Tony C. Smith (D), and Dennis J. Clouthier (iD)

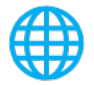

\section{ARTICLES YOU MAY BE INTERESTED IN}

Rovibrational analysis of $\mathrm{c}-\mathrm{SiC}_{2} \mathrm{H}_{2}$ : Further evidence for out-of-plane bending issues in correlated methods

The Journal of Chemical Physics 149, 024303 (2018); https://doi.org/10.1063/1.5043166

Perspective: Basic understanding of condensed phases of matter via packing models The Journal of Chemical Physics 149, 020901 (2018); https://doi.org/10.1063/1.5036657

Spectroscopy of gold atoms and gold oligomers in helium nanodroplets

The Journal of Chemical Physics 149, 024305 (2018); https://doi.org/10.1063/1.5026480 


\title{
Laser-induced fluorescence detection of the elusive SiCF free radical
}

\author{
Gretchen Rothschopf, ${ }^{1}$ Tony C. Smith, ${ }^{1}$ and Dennis J. Clouthier ${ }^{2, a)}$ \\ ${ }^{1}$ Ideal Vacuum Products, LLC, 5910 Midway Park Blvd. NE, Albuquerque, New Mexico 87109, USA \\ ${ }^{2}$ Department of Chemistry, University of Kentucky, Lexington, Kentucky 40506-0055, USA
}

(Received 17 May 2018; accepted 19 June 2018; published online 9 July 2018)

\begin{abstract}
The SiCF free radical has been spectroscopically identified for the first time. The radical was produced in an electric discharge jet using $\mathrm{CF}_{3} \mathrm{Si}\left(\mathrm{CH}_{3}\right)_{3}$ or $\mathrm{CF}_{3} \mathrm{SiH}_{3}$ vapor in high pressure argon as the precursor. The laser-induced fluorescence spectrum of the $\tilde{A}^{2} \Sigma^{+}-\tilde{X}^{2} \Pi$ band system in the $610-550 \mathrm{~nm}$ region was recorded and the ${ }^{2} \Pi_{3 / 2}$ spin component of the $0-0$ band was studied at high resolution. Rotational analysis gave the $B$ values for the combining states, and by fixing the CF bond lengths at $a b$ initio values we obtained $\mathrm{r}^{\prime \prime}(\mathrm{Si}-\mathrm{C})=1.692(1) \AA$ and $\mathrm{r}^{\prime}(\mathrm{Si}-\mathrm{C})=1.594(1) \AA$. The bond lengths correspond to a silicon-carbon double bond in the ground state and an unusual $\mathrm{Si}-\mathrm{C}$ triple bond in the excited state. Single vibronic level emission spectra yielded the ground state bending and stretching energy levels. These were fitted to a Renner-Teller model that included spin-orbit and limited vibrational anharmonicity effects. Published by AIP Publishing. https://doi.org/10.1063/1.5040473
\end{abstract}

\section{INTRODUCTION}

In 2000 , we identified the spectrum of the jet-cooled $\mathrm{SiCH}$ free radical for the first time. ${ }^{1}$ This radical turned out to be quite interesting from several points of view. By detailed rotational analysis, we showed that $\mathrm{SiCH}$ has a siliconcarbon double bond in the ground state but this distance contracts by almost $0.1 \AA$ on electronic excitation, forming a triple bond in the excited state. This odd occurrence is the result of promotion of a $\sigma$ nonbonding electron from the ground state $\mathrm{MO}$ configuration $\ldots \sigma_{\mathrm{b}}^{2} \sigma_{\mathrm{nb}}^{2} \pi^{3}$ to form an excited state $\ldots \sigma_{\mathrm{b}}^{2} \sigma_{\mathrm{nb}}^{1} \pi^{4}$ configuration with 6 electrons in a $\sigma^{2} \pi^{4}$ triple bond. $\mathrm{SiCH}$ also has a ${ }^{2} \prod$ ground state which has significant spin-orbit, Renner-Teller, and Fermi resonance complications. $^{2}$

Subsequently, we discovered the spectrum of $\mathrm{GeCH}$ which gave the first measure of the length of a germaniumcarbon triple bond. ${ }^{3}$ The spin-orbit coupling constant in the Ge molecule is large $\left(-388.3 \mathrm{~cm}^{-1}\right)$ and this leads to unusual complications in the Renner-Teller effect. ${ }^{4}$ The lower $\mu^{2} \Sigma_{1 / 2}$ component of the $(0,1,0)$ level is very close to the upper ${ }^{2} \prod_{1 / 2}$ spin-orbit component of $(0,0,0)$ and since they have the same value of $P=\Lambda+l$, they can interact, repelling each other through a process called Sears resonance. We were able to fit all of the observed ground state levels by expanding the Renner-Teller matrices to include such resonances.

In other work, we collaborated with Professor T. Steimle of Arizona State University to measure the dipole moments of $\mathrm{SiCH}$ and $\mathrm{GeCH} .{ }^{5,6}$ Although $\mathrm{SiCH}$ is likely to be an important interstellar molecule, our measured ground state dipole moment of 0.066(2) D suggests that microwave and radioastronomy studies will be quite challenging. $\mathrm{GeCH}$ has measured dipole moments of $\mu\left(X^{2} \Pi\right)=0.122(2) \mathrm{D}$ and

a)Author to whom correspondence should be addressed: dclaser@uky.edu.
$\mu\left(\tilde{A}^{2} \Sigma^{+}\right)=1.29(2) \mathrm{D}$ and the observed proton magnetic hyperfine splittings gave an upper state Fermi contact parameter of $b_{F}=163(2) \mathrm{MHz}$.

In 2002, Evans and Clouthier used ab initio theory to predict the ground and excited state properties of the unknown silicon halomethylidyne ( $\mathrm{SiCX} ; \mathrm{X}=\mathrm{F}, \mathrm{Cl}, \mathrm{Br}$ ) free radicals. ${ }^{7}$ The ground state theoretical spin-orbit coupling constants, Renner parameters, and vibrational frequencies were used to generate energy level diagrams for the $\tilde{X}^{2} \prod$ states as a guide to future experimental emission studies. In addition, the excited state excitation energies, molecular structures, and vibrational frequencies were used to simulate expected band contours for the $0-0$ bands of the radicals under typical jet-cooled conditions. Subsequently, Smith, Evans, and Clouthier ${ }^{8}$ used these theoretical predictions to help find the spectrum of the $\mathrm{SiCCl}$ free radical and showed that the ab initio results agreed well with experiment.

Our theoretical predictions ${ }^{7}$ suggested that $\mathrm{SiCF}$ would be a good target for future studies, with a significant RennerTeller effect in the ground state, a silicon-carbon triple bond in the excited state, no isotope complications, and resolvable rotational structure at high resolution. Over the intervening 15 years, we have made several attempts to record the spectrum of $\mathrm{SiCF}$, but it has turned out to be much more elusive than expected. In the present work, we have finally succeeded in detecting SiCF through the $\tilde{A}^{2} \Sigma^{+}-\tilde{X}^{2} \Pi$ LIF spectrum, have rotationally resolved the $0-0$ band, have derived approximate ground and excited state molecular structures, and have obtained sufficiently resolved emission spectra to study the Renner-Teller effect in some detail.

\section{EXPERIMENT}

The SiCF reactive intermediate was generated by seeding the vapor of a suitable precursor into high pressure argon and subjecting pulses of this gas mixture to an electric discharge. 
As described in detail elsewhere, ${ }^{9,10}$ a pulsed molecular beam valve (General Valve, series 9) injected the precursor mixture into a flow channel where an electric discharge between two stainless steel ring electrodes fragmented the precursor, producing the species of interest and a variety of other products. The reactive intermediates were rotationally and vibrationally cooled by free jet expansion into vacuum at the exit of the pulsed discharge apparatus. A $1.0 \mathrm{~cm}$ long reheat tube ${ }^{11}$ added to the end of the discharge apparatus increased production of the $\mathrm{SiCF}$ radical and suppressed the background glow from excited argon atoms.

Moderate resolution $\left(0.1 \mathrm{~cm}^{-1}\right)$ survey LIF spectra were recorded using a neodymium: yttrium aluminum garnet (Nd:YAG) pumped dye laser (Lumonics HD-500) excitation source. The fluorescence was collected by a lens, focused through appropriate longwave pass filters, onto the photocathode of a photomultiplier tube (RCA C31034A). The spectra were calibrated with optogalvanic lines from various argon- and neon-filled hollow cathode lamps to an estimated absolute accuracy of $0.1 \mathrm{~cm}^{-1}$. The laser-induced fluorescence and calibration spectra were digitized and recorded simultaneously on a homebuilt computerized data acquisition system.

Higher resolution $\left(\sim 0.015 \mathrm{~cm}^{-1}\right.$ FWHM) LIF spectra were obtained using a pulsed amplified ring dye laser (Coherent 699-21) as the excitation source using R590 dye. The linewidths in the LIF spectra were larger than that of the pulse amplified laser $\left(\sim 0.005 \mathrm{~cm}^{-1}\right)$, primarily due to power broadening because of the necessity to use substantial laser intensity to obtain recordable LIF signals. Residual Doppler effects in the unskimmed beam also contributed to the linewidth. The amplifier was a Nd:YAG $(532 \mathrm{~nm})$ pumped 4-cell system of our own design which was operated with a mixture of R620 and R640 dyes. Absolute wavelength calibration of the $\mathrm{CW}$ ring laser, to an estimated accuracy of $\sim 0.001 \mathrm{~cm}^{-1}$, was performed by simultaneously recording the sub-Doppler absorption spectrum of a heated $\mathrm{I}_{2}$ cell. ${ }^{12}$ The IodineSpec5 program was used to calculate the hyperfine transition frequencies. ${ }^{13}$ Frequency calibration between the iodine lines was obtained by interpolation between transmission fringes through a pressure-stabilized confocal etalon (free spectral range FSR $=1.5 \mathrm{GHz}$ ) whose cavity length was actively locked to a frequency stabilized $\mathrm{He}-\mathrm{Ne}$ laser.

For emission spectroscopy, previously measured LIF band maxima in the SiCF spectra were excited by the dye laser and the resulting fluorescence was imaged with $f / 4$ optics onto the entrance slit of a $0.5 \mathrm{~m}$ scanning monochromator (Spex $500 \mathrm{M})$. The pulsed fluorescence signals were detected with a gated CCD camera (Andor iStar 320T) and recorded digitally. The emission spectra were calibrated to an estimated accuracy of $\pm 1 \mathrm{~cm}^{-1}$ using emission lines from a neon lamp. A 1200 line/mm grating blazed at $750 \mathrm{~nm}$ was employed in this work, which gave a bandpass of $29.9 \mathrm{~nm}$ with $18 \mathrm{~mm}$ effective active area on the CCD. A spectral resolution of $0.2 \mathrm{~nm}$ was typical, depending on the signal intensity.

In order to untangle the weak SiCF LIF bands from the many impurity features, the emission setup was used in 2D-LIF mode, in which an emission spectrum was recorded for every laser excitation wavenumber. A lower resolution 300 line/mm grating (blazed at $750 \mathrm{~nm}$ ) was used with the gated CCD detector, the laser was incremented in steps of $0.2 \mathrm{~cm}^{-1}$, and 200 shots were averaged for each laser step. The $120 \mathrm{~nm}$ detection region of the low resolution grating/CCD combination allowed us to record the major features of each emission spectrum without moving the grating. The LIF and emission wavenumbers were calibrated as before. Software was written to extract LIF spectra from user selected segments of the recorded emission spectra, effectively isolating the LIF bands of SiCF.

Trifluoromethyltrimethylsilane $\left[\mathrm{CF}_{3} \mathrm{Si}\left(\mathrm{CH}_{3}\right)_{3}\right.$, Aldrich, 95\%], as received, was transferred to a Pyrex U-tube, degassed, pressured with 40-60 psi of argon, cooled to $-15^{\circ} \mathrm{C}$, and the gaseous mixture was used as a SiCF precursor. In addition, three other possible precursors were synthesized. Liquid (trifluoromethyl)trichlorosilane $\left(\mathrm{CF}_{3} \mathrm{SiCl}_{3}\right)$ was obtained by the reaction of a $\mathrm{CF}_{3} \mathrm{Br}+\mathrm{P}\left(\mathrm{NEt}_{2}\right)_{3}$ complex with $\mathrm{SiCl}_{4}$ in benzonitrile according to the method of Beckers et al. ${ }^{14} \mathrm{CF}_{3} \mathrm{SiCl}_{3}$ was fluorinated by reaction with $\mathrm{SbF}_{3}$ to produce gaseous (trifluoromethyl)trifluorosilane $\left(\mathrm{CF}_{3} \mathrm{SiF}_{3}\right)$ or reduced with $\mathrm{LiAlH}_{4}$ to yield gaseous trifluoromethylsilane $\left(\mathrm{CF}_{3} \mathrm{SiH}_{3}\right){ }^{15}$ The purity of all three compounds was checked by gas phase infrared spectroscopy. ${ }^{14,15}$ These reactive compounds were stored at low temperatures in Pyrex U-tubes, pressurized with 40-60 psi of argon and delivered to the pulsed valve through stainless steel tubing.

\section{RESULTS AND ANALYSIS}

\section{A. LIF spectra}

Our previous theoretical study ${ }^{7}$ predicted the ${ }^{2} \prod_{3 / 2}$ spin component of the $0-0$ band of SiCF to appear with an intense Q-branch at $\sim 17104 \mathrm{~cm}^{-1}$ [CCSD(T)/aug-cc-pVTZ] accompanied by a weaker transition from the upper spin-orbit component $\left({ }^{2} \prod_{1 / 2}\right) 80-93 \mathrm{~cm}^{-1}$ to lower energy. Under our typical experimental conditions, the LIF bands were predicted to be $15-20 \mathrm{~cm}^{-1}$ wide with possibly resolved rotational lines at either extreme. Armed with these expectations, we set out to search for the LIF spectrum using the commercially available $\mathrm{CF}_{3} \mathrm{Si}\left(\mathrm{CH}_{3}\right)_{3}$ precursor. As shown in Fig. 1, this compound gives a very rich LIF spectrum in the target region, which our experience allowed us to readily identify as the spectra of HCF, $\mathrm{SiH}_{2}$, and $\mathrm{SiCH}_{2}$. However, among the weaker features, there were a few unidentified bands which matched our expectations for SiCF. Emission spectra (vide infra) of these bands were also promising, showing a pair of bands 665 and $770 \mathrm{~cm}^{-1}$ to the red of the excitation laser, giving a vibrational interval of $665 \mathrm{~cm}^{-1}$ and a splitting of $105 \mathrm{~cm}^{-1}$, comparable to our ab initio predictions of $v_{3}^{\prime \prime}=685-639 \mathrm{~cm}^{-1}$ and spin-orbit coupling constant of $A=-80$ to $-93 \mathrm{~cm}^{-1}$. The pattern of bands suggested the $0-0$ band should occur at lower energies, so we moved one dye to the red and recorded the spectrum shown in Fig. 2. The prominent LIF band at the center of the spectrum with Q-branch at $16571 \mathrm{~cm}^{-1}$ we assign as the ${ }^{2} \Sigma^{+}-{ }^{2} \Pi_{3 / 2}$ spinorbit component of the $0-0$ band of SiCF. Unfortunately, it is partially overlapped by known bands of the $\tilde{A}^{1} \mathrm{~A}_{2}-\tilde{X}^{1} \mathrm{~A}_{1}$ system of $\mathrm{SiCH}_{2}$ which we studied many years ago. ${ }^{16}$ 


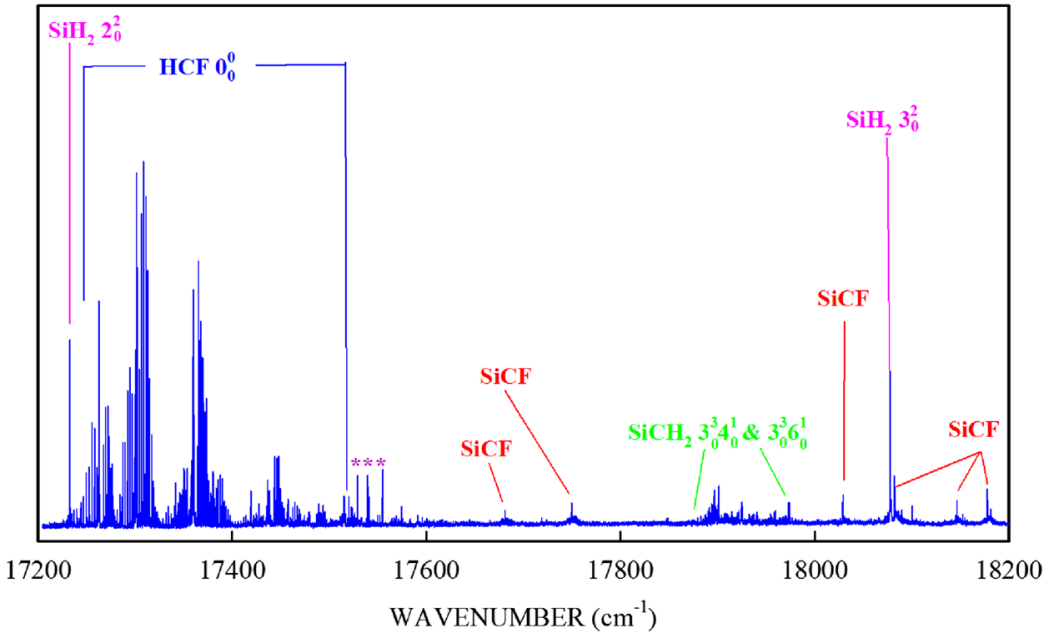

FIG. 1. A low resolution LIF spectrum of the products of an electrical discharge through $\mathrm{CF}_{3} \mathrm{Si}\left(\mathrm{CH}_{3}\right)_{3}$ vapor diluted in argon. Most of the bands can be attributed to $\mathrm{SiH}_{2}, \mathrm{HCF}$, and $\mathrm{SiCH}_{2}$ but several weak bands with prominent Q-branches can be assigned as due to the $\mathrm{SiCF}$ free radical.
In an effort to clean up the spectrum, get rid of a lot of the extraneous impurity bands, and improve the LIF signal, we went to considerable trouble to synthesize alternate precursors which did not contain hydrogen. Of these, the most promising was $\mathrm{CF}_{3} \mathrm{SiF}_{3}$ which would seem ideally suited to produce SiCF. However, we could not find any experimental conditions which would give $\mathrm{SiCF}$ signals from this molecule, despite substantial effort. Mystified, we tried $\mathrm{CF}_{3} \mathrm{SiCl}_{3}$, which we had in hand since it is the precursor to $\mathrm{CF}_{3} \mathrm{SiF}_{3}$. The result was the same-no $\mathrm{SiCF}$ bands were observed. At this point, we theorized that the strong $\mathrm{Si}-\mathrm{F}(135 \mathrm{kcal} / \mathrm{mol})$, $\mathrm{C}-\mathrm{F}(116 \mathrm{kcal} / \mathrm{mol})$ and $\mathrm{Si}-\mathrm{Cl}(90 \mathrm{kcal} / \mathrm{mol})$ bonds ${ }^{17}$ in these molecules were hampering the discharge from producing SiCF. In $\mathrm{CF}_{3} \mathrm{SiCl}_{3}$ and $\mathrm{CF}_{3} \mathrm{SiF}_{3}$ with the plethora of electron withdrawing groups, the silicon-carbon bond is the weak link and they preferentially produce $\mathrm{CF}_{3}+\mathrm{SiX}_{3}$ or perhaps $\mathrm{CF}_{2}+\mathrm{SiX}_{4}$ as is observed thermally. ${ }^{18} \mathrm{In}_{3} \mathrm{CF}_{3} \mathrm{Si}\left(\mathrm{CH}_{3}\right)_{3}$, the $\mathrm{Si}-\mathrm{CH}_{3}$ bonds are closer in energy to $\mathrm{CF}_{3}-\mathrm{Si}$, so there is a small but finite chance of producing $\mathrm{SiCF}$, as is observed experimentally. So we theorized that we needed a $\mathrm{CF}_{3} \mathrm{SiX}_{3}$ compound with readily eliminated $\mathrm{X}$ substituents. We settled on $\mathrm{CF}_{3} \mathrm{SiH}_{3}$ ( $\mathrm{Si}-\mathrm{H}$ bond strength $\sim 75 \mathrm{kcal} / \mathrm{mol}$ ) which was easily made by reducing $\mathrm{CF}_{3} \mathrm{SiCl}_{3}$. The result, shown in Fig. 2, was positive- $\mathrm{SiCF}$ is made in a discharge through trifluoromethylsilane, the impurity spectra are different, but unfortunately the signals remained weak and rather unstable.

With two different precursors in hand, we continued experiments to prove that we had observed SiCF for the first time. Figure 3 shows the LIF spectrum of the $0-0$ band in comparison to that calculated from our $a b$ initio structures. ${ }^{7}$ It is clear that the observed band contour matches the prediction rather well, and the Q-branch is only $\sim 530 \mathrm{~cm}^{-1}$ lower in energy than our theoretical prediction, both factors providing good evidence that the spectra are due to $\mathrm{SiCF}$.

Despite our substantial efforts at precursor development, the LIF spectra continued to be weak and overlapped by impurity bands. These difficulties were overcome using 2D-LIF spectroscopic techniques. Figure 4 shows an example of our success in extracting the positions of the $3{ }_{0}^{1}$ and $2{ }_{0}^{2}$ bands from

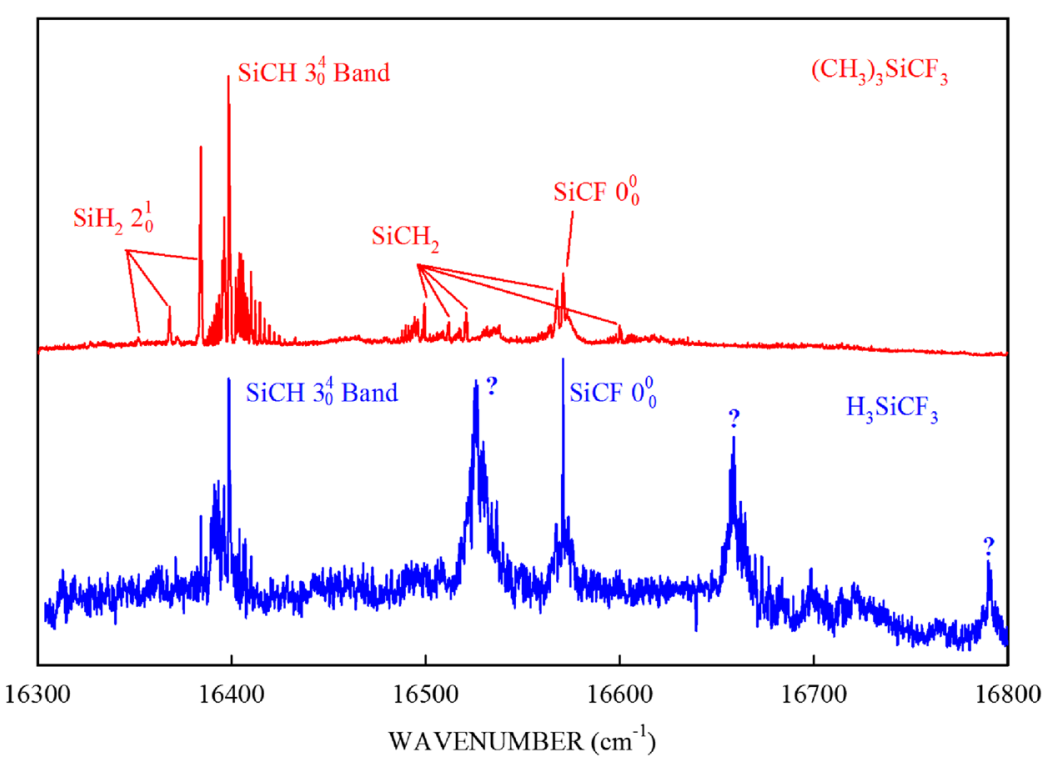

FIG. 2. Low resolution LIF spectra obtained with the $\mathrm{CF}_{3} \mathrm{Si}\left(\mathrm{CH}_{3}\right)_{3}$ precursor (top) and the $\mathrm{CF}_{3} \mathrm{SiH}_{3}$ precursor (bottom). Bands marked? are due to an unidentified impurity. 


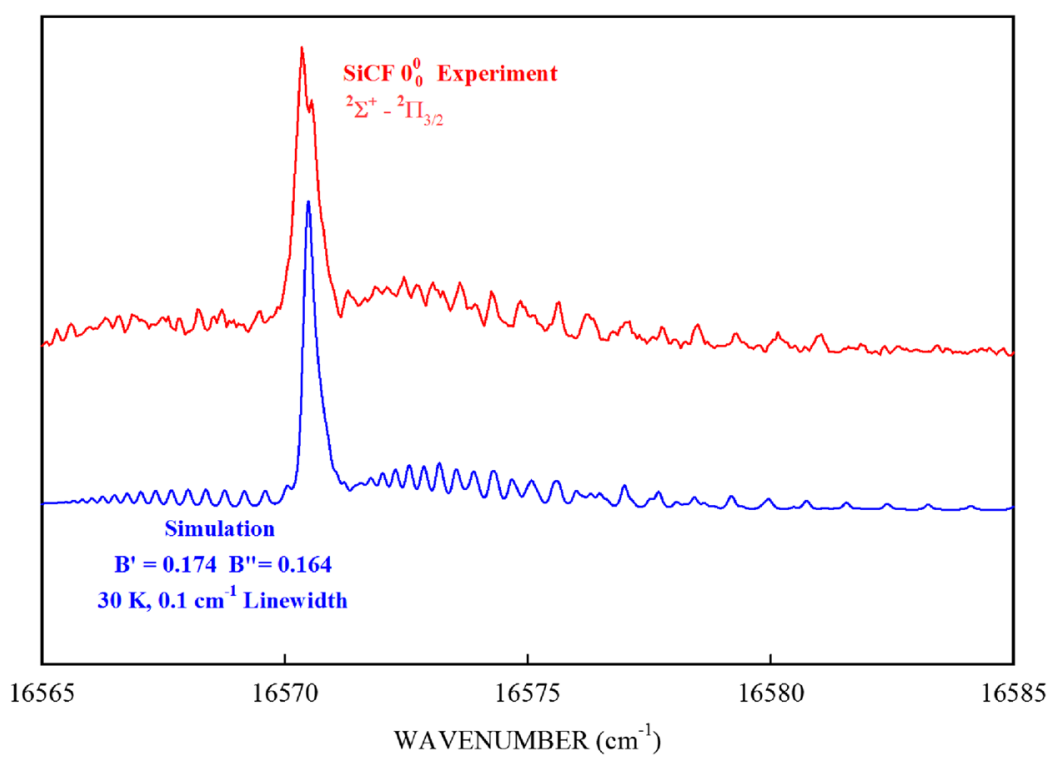

FIG. 3. A medium resolution scan of the $0-0$ band of $\mathrm{SiCF}$ [top, $\mathrm{CF}_{3} \mathrm{Si}\left(\mathrm{CH}_{3}\right)_{3}$ precursor] and our simulation of the band contour based on the $a b$ initio geometries from Ref. 7.

the much stronger HCF features in this region (see Fig. 1). The bottom portion of Fig. 4 shows the 2D-LIF data as a color keyed plot with the excitation laser wavenumber $\left(\mathrm{cm}^{-1}\right)$ along the abscissa and the fluorescence displacement (laser wavenumber-emission wavenumber) along the ordinate. The fluorescence intensity is represented by color, with black being the most intense, fading through red, yellow, green, blue, and then white for the least intense, as shown by the color intensity scale along the right-hand side of the figure. The most intense black/bright red emission bands occur at 0 and $1410 \mathrm{~cm}^{-1}$ displacement and are readily assigned as HCF emission down to the $0_{0}$ and $2{ }_{1}$ levels. In the present case, the much weaker $\mathrm{SiCF}$ emission spectra occur in regions between the HCF bands, predominantly from 490 to $700 \mathrm{~cm}^{-1}\left(2_{2}\right.$ band components at 492 and $644 \mathrm{~cm}^{-1}$, vide infra) and 1700 to $2200 \mathrm{~cm}^{-1}\left(2{ }_{2} 3_{2}\right.$ components). By setting these filter regions in software, the top SiCF LIF spectrum, substantially free of HCF features, was obtained and the bands were assigned based on their excited state intervals and emission spectra. Comparing Figs. 1 and 4, there is little evidence of these weak SiCF bands in the total LIF spectrum but they are clearly apparent in the 2D scan. The 2D-LIF technique is a powerful tool which makes it possible to identify weak fluorescing species in regions where the spectrum is congested with overlapping band systems. Table I summarizes the Q-branch measurements and assignments for the observed SiCF LIF bands. The list is still somewhat limited due to the weakness of the spectra and persistent problems with overlapping impurity bands. We note that some bands in the table are formally forbidden, such as $2_{0}^{1}$. The occurrence of such vibronically induced bands following the selection rule $\Delta v_{2}= \pm 1$ is not unexpected, as they are also prominent in the spectra of $\mathrm{SiCH}^{1}$ and $\mathrm{SiCCl}^{8}$

\section{B. High resolution rotationally resolved spectra}

Although our observed SiCF 0-0 LIF band is very weak, we fortunately were able to obtain a high resolution spectrum of the ${ }^{2} \prod_{3 / 2}$ component. This involved concatenating a large number of individual $1 \mathrm{~cm}^{-1}$ ring laser scans, each individually

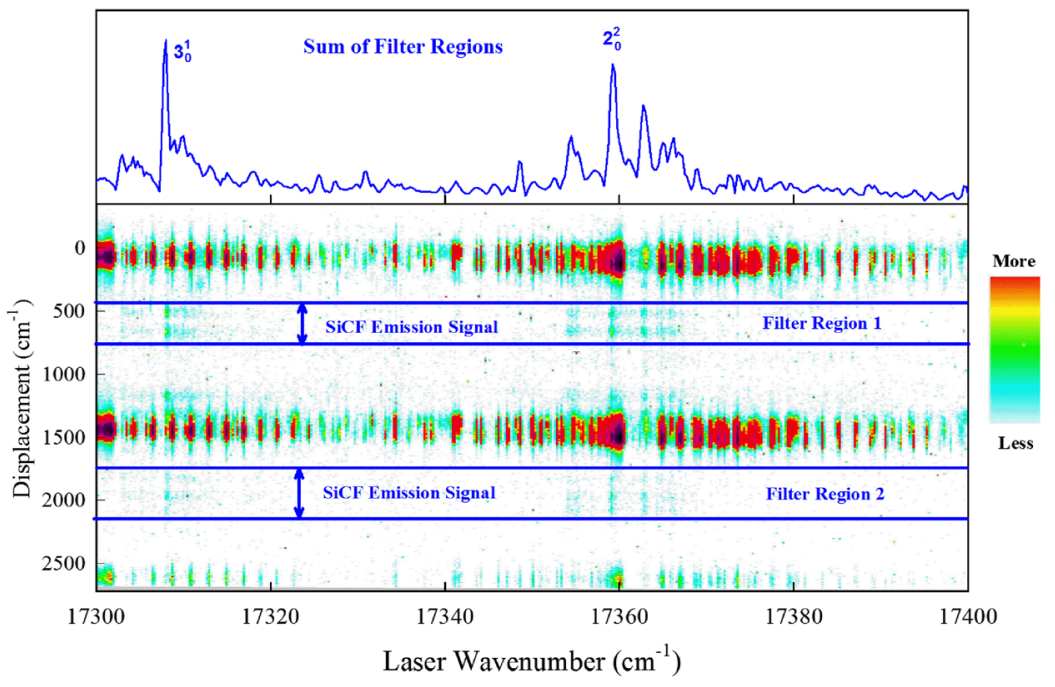

FIG. 4. A 2D-LIF spectrum of $\mathrm{SiCF}\left[\mathrm{CF}_{3} \mathrm{Si}\left(\mathrm{CH}_{3}\right)_{3}\right.$ precursor] in the $17300-17400 \mathrm{~cm}^{-1}$ region. The excitation wavenumber of the laser is given along the abscissa and the emission spectrum at each laser position is given along the ordinate. The emission spectra are plotted as displacement from the laser position, which gives a direct measure of the ground state energy of each transition, and the intensities are color coded (see the legend on the RHS). By setting two filter regions and combining their intensities in software, the 2D-LIF spectrum of SiCF (top trace) was obtained, almost completely eliminating the impurity emission from HCF (see Fig. 1). 
TABLE I. Assignments and Q-branch maxima of the observed bands (all values in $\mathrm{cm}^{-1}$ ) in the LIF spectrum of SiCF.

\begin{tabular}{lccc}
\hline \hline Assign. & ${ }^{2} \Pi_{3 / 2}$ & ${ }^{2} \Pi_{1 / 2}$ & Comment \\
\hline $0_{0}^{0}$ & 16571.0 & 16465.8 & $\Delta_{\text {so }}=-105.2$ \\
$2_{0}^{1}$ & 16958.9 & 16853.7 & $\Delta_{\text {so }}=-105.2 v_{2}^{\prime}=387.9$ \\
$3_{0}^{1}$ & 17308.1 & $\ldots$ & $v_{3}^{\prime}=737.1$ \\
$2_{0}^{2}$ & 17359.2 & $\ldots$ & $2_{0}^{1}+400.3$ \\
$2_{0}^{1} 3_{0}^{1}$ & 17680.9 & $\ldots$ & $2_{0}^{1}+722.0$ \\
$2_{0}^{3}$ & 17749.7 & $\ldots$ & $2_{0}^{2}+390.5$ \\
$3_{0}^{2}$ & 18029.2 & $\ldots$ & $3_{0}^{1}+721.1$ \\
$2_{0}^{2} 3_{0}^{1}$ & 18082.3 & $\ldots$ & $2_{0}^{2}+723.1$ \\
$2_{0}^{4}$ & 18146.5 & $\ldots$ & $2_{0}^{3}+396.8$ \\
$1_{0}^{1}$ & 18177.9 & $\ldots$ & $v_{1}^{\prime}=1606.9$ \\
\hline \hline
\end{tabular}

calibrated, to obtain the spectrum shown in Fig. 5. Although the central $Q$ branch features are not well-resolved, the branches on either side show clearly delineated rotational lines. Figure 6 shows a small portion of the high resolution spectrum in the region near the onset of the $R_{21}$ branch. The $J$-dependent upper state spin-rotation splittings evident as the difference of the $Q_{21}\left(J^{\prime \prime}\right)$ and $R_{11}\left(J^{\prime \prime}\right)$ lines are also illustrated.

We have fitted the rotational structure of our spectrum using the very convenient graphical PGopher program. ${ }^{19,20}$ Since only one spin component was observed, we fixed the lower state spin-orbit coupling constant $A$ at the value of $-105.2 \mathrm{~cm}^{-1}$ obtained from the low resolution data (Table I). Our starting point was to use the predicted rotational constants obtained in our previous $a b$ initio work $^{7}$ to simulate the spectrum (see Fig. 3) which allowed us to assign lines in the resolved branches with relative ease. Initially, we only fitted the two $B$ values and the band origin, adding the excited state spin-rotation constant $\gamma$ as needed to fit the higher $J^{\prime} Q_{21}$ and $R_{11}$ lines. It was soon evident that the

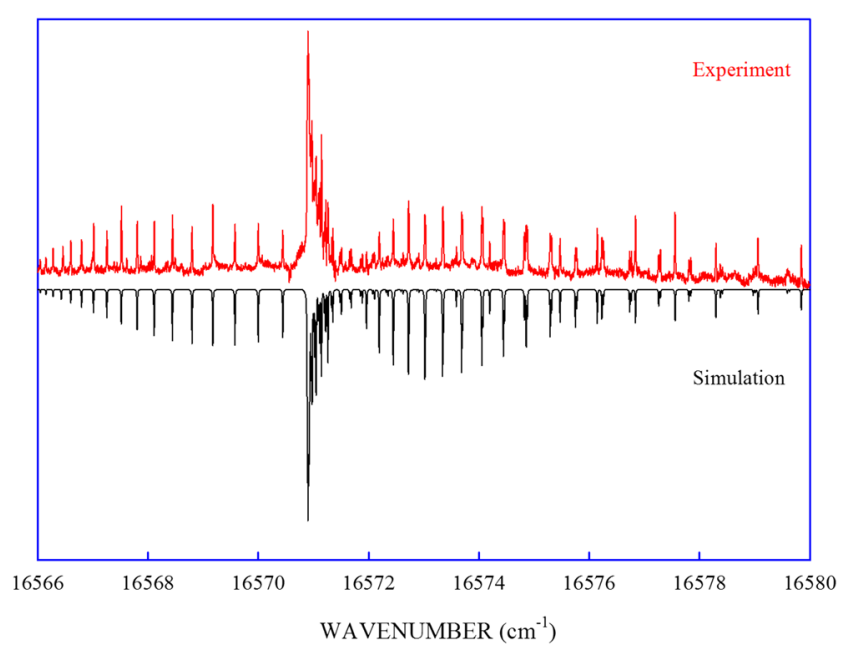

FIG. 5. The high resolution spectrum of the ${ }^{2} \Pi_{3 / 2}$ component of the $0-0$ band of $\mathrm{SiCF}$ [top, $\mathrm{CF}_{3} \mathrm{Si}\left(\mathrm{CH}_{3}\right)_{3}$ precursor] and the simulation of the spectrum at a rotational temperature of $20 \mathrm{~K}$ (bottom). The experimental spectrum was obtained by concatenating 15 individual $1 \mathrm{~cm}^{-1}$ scans with varying laser power and experimental conditions, accounting for the irregularity in the baseline. Some weaker features in the spectra not reproduced in the simulation are due to impurity fluorescence.

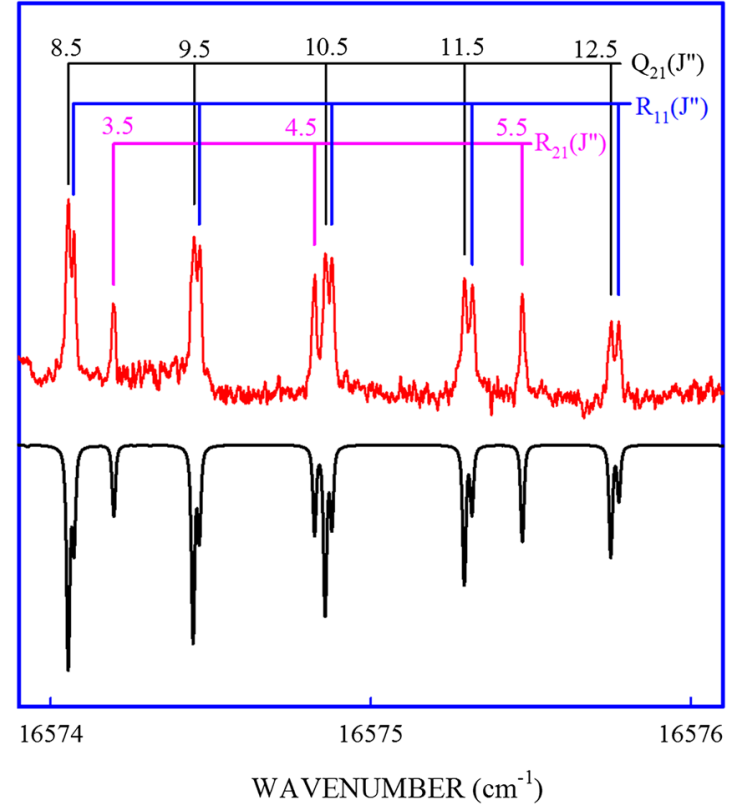

FIG. 6. A small portion of the high resolution spectrum of the ${ }^{2} \Pi_{3 / 2}$ component of the $0-0$ band of $\mathrm{SiCF}$ [top, $\mathrm{CF}_{3} \mathrm{Si}\left(\mathrm{CH}_{3}\right)_{3}$ precursor] showing the onset of resolved spin-rotation splittings $\left[\mathrm{R}_{11}\left(\mathrm{~J}^{\prime \prime}\right)-\mathrm{Q}_{21}\left(\mathrm{~J}^{\prime \prime}\right)\right]$ and the $\mathrm{R}_{21}$ branch lines. The simulation (bottom) was obtained using the constants in Table III and a rotational temperature of $20 \mathrm{~K}$.

ground state $\Lambda$ doubling was negligible at our resolution and that the centrifugal distortion constants $(D)$ were not determinable in either state. In the final analysis, we used 5 constants to fit 68 transitions to an overall standard deviation of $0.0015 \mathrm{~cm}^{-1}$, with the results summarized in Tables II and III. The $P_{11}(15.5)$ line was left out of the analysis and it is not listed in Table II, as it was found that the scan segment had a mode hop which invalidated the line frequency determination. Simulations of the spectra using the fitted constants and an estimated rotational temperature of $20 \mathrm{~K}$ are shown in Figs. 5 and 6, illustrating good agreement with the line positions and reasonable reproduction of the relative intensities. No perturbations were detected in any of the branches of the spectrum.

\section{Emission spectra}

By extensive signal averaging (20 000 laser shots), we were able to obtain good emission spectra from laser excitation of the ${ }^{2} \prod_{3 / 2}$ spin-orbit components of the $0_{0}^{0}$ and $2_{0}^{1}$ bands, as illustrated in Fig. 7. The figure also shows the calculated energy level pattern for the ground state, derived from our previous $a b$ initio study. ${ }^{7}$ The $0-0$ band emission spectrum is quite simple, consists of transitions down to the two spin-orbit components of the zero-point level (the gated CCD allows us to gate out all of the scattered laser light), a second doublet at $666 / 770 \mathrm{~cm}^{-1}$ assigned as $3_{1}^{0}$, and a similar $3_{2}^{0}$ band at $1334 / 1431 \mathrm{~cm}^{-1}$. The spin-orbit intervals are $105 \mathrm{~cm}^{-1}(000)$, $104 \mathrm{~cm}^{-1}(001)$, and $97 \mathrm{~cm}^{-1}(002)$. As is evident from the figure, the smaller spin-orbit splitting in (002) is readily explained by a Fermi resonance interaction with the nearby (021) level, accounting for the intensity of the transition to the latter in the emission spectrum. We were also able to assign transitions to 
TABLE II. Rotational line frequencies $\left(\mathrm{cm}^{-1}\right)$ and assignments for the ${ }^{2} \Sigma^{+}-{ }^{2} \Pi_{3 / 2}$ component of the $0-0$ band of SiCF.

\begin{tabular}{|c|c|c|c|c|c|c|}
\hline $\mathrm{J}^{\prime \prime}$ & $\mathrm{R}_{21}$ & $\mathrm{R}_{11}$ & $\mathrm{Q}_{21}$ & $\mathrm{Q}_{11}$ & $\mathrm{P}_{21}$ & $\mathrm{P}_{11}$ \\
\hline 1.5 & & $* 16571.9629(-30)^{\mathrm{a}}$ & *16571.9629(18) & & & \\
\hline 2.5 & $16573.5943(17)$ & $* 16572.1938(-52)$ & *16572.1938(16) & & & $16570.4431(-14)$ \\
\hline 3.5 & $16574.1967(-1)$ & $* 16572.4486(-60)$ & *16572.4486(26) & & & $16569.9992(-2)$ \\
\hline 4.5 & $16574.8237(-1)$ & $* 16572.7257(-73)$ & *16572.7257(32) & & & $16569.5757(-11)$ \\
\hline 5.5 & $16575.4738(6)$ & $* 16573.0250(-90)$ & *16573.0250(34) & & & $16569.1749(-21)$ \\
\hline 6.5 & $16576.1467(-12)$ & $* 16573.3481(-97)$ & *16573.3481(47) & & & $16568.7992(-7)$ \\
\hline 7.5 & $16576.8406(2)$ & $* 16573.6938(-104)$ & *16573.6938(59) & & & $16568.4443(-12)$ \\
\hline 8.5 & $16577.5579(-2)$ & $16574.0710(-18)$ & $16574.0549(1)$ & & & $16568.1140(2)$ \\
\hline 9.5 & $16578.2991(6)$ & $16574.4636(-11)$ & $16574.4483(35)$ & & & $16567.8040(-7)$ \\
\hline 10.5 & $16579.0606(-9)$ & $16574.8778(-15)$ & $16574.8601(27)$ & *16571.0245(10) & $16571.0021(-15)$ & $16567.5192(8)$ \\
\hline 11.5 & $16579.8471(-3)$ & $16575.3172(7)$ & $16575.2937(10)$ & *16571.1112(9) & *16571.0901(16) & $16567.2548(-1)$ \\
\hline 12.5 & $16580.6555(-3)$ & $16575.7739(-26)$ & $16575.7501(-7)$ & *16571.2225(27) & *16571.2023(62) & $16567.0147(8)$ \\
\hline 13.5 & $16581.4880(10)$ & $16576.2599(8)$ & $16576.2315(-1)$ & $16571.3546(24)$ & $16571.3288(22)$ & $16566.7952(-5)$ \\
\hline 14.5 & $16582.3389(-20)$ & $16576.7659(14)$ & $16576.7347(-10)$ & $16571.5053(-18)$ & $16571.4802(6)$ & $16566.5997(-5)$ \\
\hline 15.5 & & $16577.2928(3)$ & $16577.2602(-9)$ & $16571.6831(-17)$ & $16571.6554(1)$ & $\ldots$ \\
\hline 16.5 & & $16577.8448(16)$ & $16577.8108(8)$ & $16571.8855(4)$ & $16571.8550(12)$ & $16566.2785(12)$ \\
\hline 17.5 & & $16578.4190(23)$ & $16578.3803(-13)$ & $16572.1050(-31)$ & $16572.0754(-5)$ & $16566.1496(-4)$ \\
\hline 18.5 & & $16579.0144(15)$ & $16578.9731(-27)$ & $16572.3539(-2)$ & $16572.3209(22)$ & $16566.0476(24)$ \\
\hline 19.5 & & & & $16572.6253(30)$ & $16572.5857(4)$ & $16565.9627(-6)$ \\
\hline 20.5 & & & & $16572.9106(-28)$ & $16572.8717(-28)$ & \\
\hline
\end{tabular}

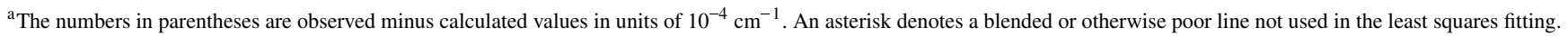

both spin-orbit components of the $1_{1}^{0}$ band at the very end of the spectrum.

The $2{ }_{0}^{1}$ band emission spectrum is almost completely different, which is expected from the selection rules and fortunate as the observed transitions basically define the Renner-Teller problem. There is weak resonance fluorescence down to the $(000)^{2} \prod_{3 / 2}$ level, coincident with the laser wavelength, and then a strong group of four lines down to (010) which satisfy the $\Delta v_{2}=0$ selection rule. This pattern is repeated for transitions down to (011).

We have fitted our data to a Renner-Teller model including spin-orbit and Fermi resonance complications using a computer program ${ }^{21}$ written especially for this purpose and employed previously to analyze similar emission data for $\mathrm{SiCH}^{2}{ }^{2} \mathrm{GeCH},{ }^{4}$ and $\mathrm{SiCCl}^{8}$ We were able to fit the 18 observed ground state energy levels with a minimal set of constants including $\omega_{1}, \omega_{2}, \omega_{3}, \varepsilon \omega_{2}, g_{k}, A$ and the $x_{23}$ anharmonicity constant, to an overall standard deviation of $2.24 \mathrm{~cm}^{-1}$, which is very satisfactory for a Renner-Teller analysis. Although there is evidence of Fermi resonance in the spectrum, neither of the Fermi resonance parameters $W_{1}$ or $W_{2}$ were determinable by least squares due to an insufficiency

TABLE III. The molecular constants $\left(\right.$ in $\mathrm{cm}^{-1}$ ) of SiCF.

\begin{tabular}{lcc}
\hline \hline Parameter & ${ }^{2} \Pi$ & ${ }^{2} \Sigma^{+}$ \\
\hline$T_{0}$ & $\ldots$ & $16518.804_{1}(1)$ \\
$B$ & $0.164116_{2}(25)^{\mathrm{a}}$ & $0.175212_{7}(26)$ \\
$A$ & $-105.2^{\mathrm{b}}$ & $\ldots$ \\
$\gamma$ & $\cdots$ & $0.00190(9)$ \\
\hline
\end{tabular}

${ }^{\mathrm{a}}$ The numbers in parentheses are $3 \sigma$ error limits and are right-justified to the last digit on the line; sufficient additional digits are quoted to reproduce the original data to full accuracy. 68 transitions were fitted with an overall standard deviation of $0.0015 \mathrm{~cm}^{-1}$.

${ }^{\mathrm{b}}$ Value taken from Table I and fixed in the least squares fitting. of data on the higher energy levels. The fitted levels and their least squares residuals are given in Table IV and the resulting constants are summarized in Table V.

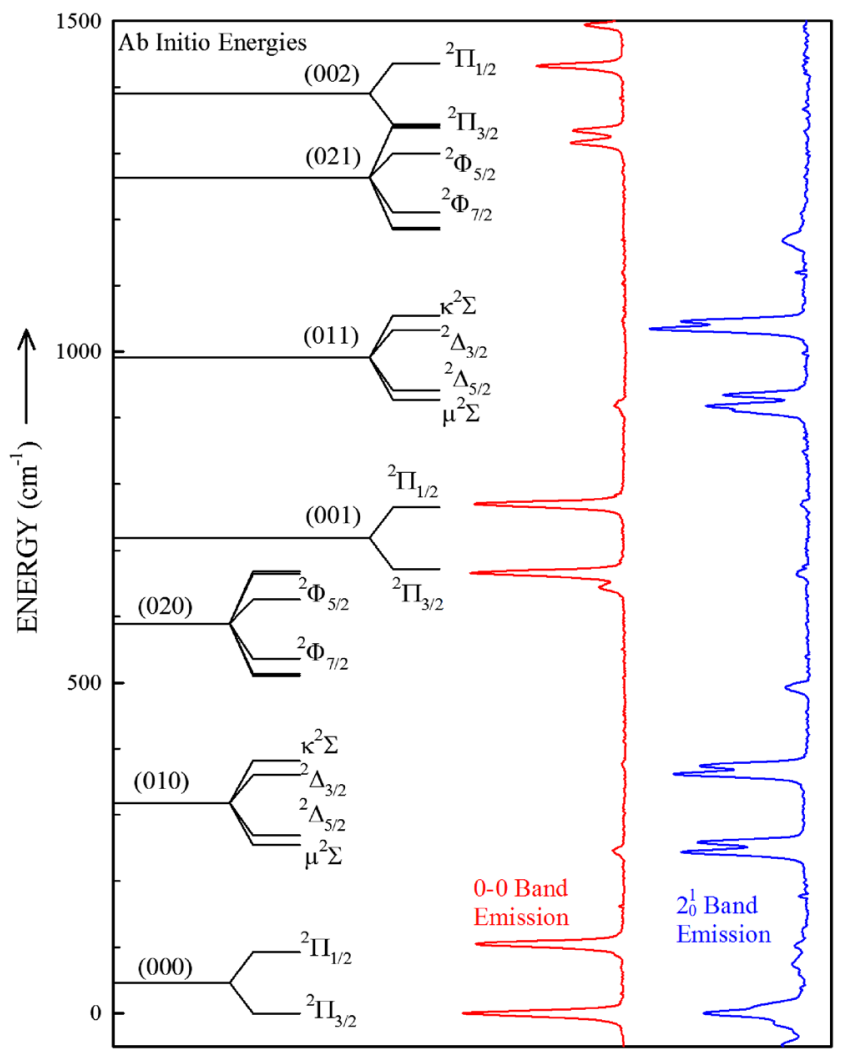

FIG. 7. The emission spectra observed from laser excitation of the ${ }^{2} \Pi_{3 / 2}$ spin-orbit components of the $0_{0}^{0}$ and $2{ }_{0}^{1}$ bands of $\mathrm{SiCF}\left[\mathrm{CF}_{3} \mathrm{Si}\left(\mathrm{CH}_{3}\right)_{3}\right.$ precursor]. The vertical scale is displacement from the excitation laser wavenumber, giving a direct measure of the relative ground state energy of each transition. The corresponding energy levels obtained from our ab initio study (Ref. 7) are given on the left-hand side. 
TABLE IV. Vibrational levels of the $\tilde{X}^{2} \Pi_{i}$ state of $\operatorname{SiCF}\left[\mathrm{in} \mathrm{cm}^{-1}\right.$ relative to the lowest (000) ${ }^{2} \Pi_{3 / 2}$ level].

\begin{tabular}{lcr}
\hline \hline State $\left(v_{1} v_{2} v_{3}\right)$ & Energy & Obs. - Calc. $^{\text {a }}$ \\
\hline$(000)^{2} \Pi_{1 / 2}$ & 105 & 1.94 \\
$(010) \mu^{2} \Sigma$ & 244 & -0.08 \\
$(010)^{2} \Delta_{5 / 2}$ & 259 & -1.33 \\
$(010)^{2} \Delta_{3 / 2}$ & 361 & -1.13 \\
$(010) \kappa^{2} \Sigma$ & 374 & 1.22 \\
$(020) \mu^{2} \Pi_{3 / 2}$ & 492 & -1.43 \\
$(020) \kappa^{2} \Pi_{3 / 2}$ & 644 & 2.10 \\
$(001) \mu^{2} \Pi_{3 / 2}$ & 666 & -0.19 \\
$(001) \kappa^{2} \Pi_{1 / 2}$ & 770 & 0.72 \\
$(011) \mu^{2} \Sigma$ & 918 & 2.16 \\
$(011)^{2} \Delta_{5 / 2}$ & 935 & 2.87 \\
$(011)^{2} \Delta_{3 / 2}$ & 1035 & 0.98 \\
$(011) \kappa^{2} \Sigma$ & 1045 & 0.44 \\
$(021) \kappa^{2} \Pi_{3 / 2}$ & 1316 & -3.23 \\
$(002){ }^{2} \Pi_{3 / 2}$ & 1334 & 1.62 \\
$(002){ }^{2} \Pi_{1 / 2}$ & 1432 & -3.50 \\
$(100)^{2} \Pi_{3 / 2}$ & 1494 & -0.47 \\
$(100)^{2} \Pi_{1 / 2}$ & 1598 & 0.47 \\
\hline \hline
\end{tabular}

${ }^{\mathrm{a}}$ From our Renner-Teller analysis of the emission spectra.

\section{DISCUSSION}

\section{A. The molecular structure of SiCF}

With only a single $B$ value for each state, it is not possible to completely determine the molecular structure of the $\mathrm{SiCF}$ radical. However, if we constrain the $\mathrm{CF}$ bond lengths to our $a b$ initio values, ${ }^{7}$ we can get approximate $\mathrm{SiC}$ bond lengths of $r^{\prime \prime}=1.692(1)$ and $r^{\prime} 1.594(1) \AA$, as summarized in Table V. Theory predicts a $0.1 \AA$ decrease in the $\mathrm{SiC}$ bond length on electronic excitation, mirrored almost exactly by the $0.098 \AA$ A value obtained from the rotational constants. Since the change in the CF bond length is predicted to be miniscule $(+0.008 \AA)$ on electronic excitation, it is unlikely that any over- or underestimation of the carbon-halogen bond length can substantially affect the experimentally derived decrease in the $\mathrm{Si}-\mathrm{C}$ bond length. It is evident that the $\mathrm{SiCF}$ bond order increases substantially on electronic excitation, precisely as found from purely experimental structures derived previously for $\mathrm{SiCH}^{1}$ and $\mathrm{GeCH}^{3}$

These results can be readily understood from the molecular orbitals (MOs) involved. In the ground state of SiCF, the electron configuration is ...( $\left.\sigma_{\mathrm{b}}\right)^{2}\left(\sigma_{\mathrm{nb}}\right)^{2}(\pi)^{3}\left({ }^{2} \Pi\right)$, where $\sigma_{\mathrm{b}}$ is an $\mathrm{Si}-\mathrm{C}$ bonding orbital, $\sigma_{\mathrm{nb}}$ is a nonbonding lone pair on the silicon atom, and the $\pi$ orbitals are $\mathrm{Si}-\mathrm{C}$ bonding. In both $\mathrm{SiCH}^{1}$ and $\mathrm{SiCF}$, the resulting $\mathrm{SiC}$ bond length is $1.693 \pm 0.001 \AA$, which can be interpreted as a carbon-silicon double bond. Clearly, halogen substitution onto $\mathrm{SiCH}$ has little effect on this bond. The first electronic excited state is formed by promotion of an electron from the nonbonding orbital to the $\pi$ bonding orbitals, yielding the configuration ... $\left(\sigma_{\mathrm{b}}\right)^{2}\left(\sigma_{\mathrm{nb}}\right)^{1}(\pi)^{4},\left({ }^{2} \Sigma^{+}\right)$which now has a total of six electrons in bonding orbitals, forming a carbon-silicon triple bond. In SiCF, the bond length is 1.594(1) $\AA$, analogous to the $1.6118(1) \AA$ bond length in excited $\mathrm{SiCH},{ }^{1}$ precisely as predicted by ab initio theory. ${ }^{7}$

We can also compare the $\mathrm{Si}-\mathrm{C}$ bond lengths of $\mathrm{SiCF}$ to those reported in the literature for other compounds. Silicon-carbon single bonds are much longer, of the order of 1.87-1.91 $\AA^{22}$ We determined the length of the silicon-carbon double bond in transient silylidene $\left(\mathrm{H}_{2} \mathrm{C}=\mathrm{Si}\right)^{23}$ to be $1.706 \AA$,

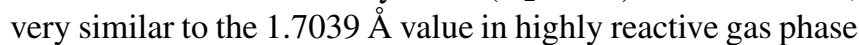
$\mathrm{H}_{2} \mathrm{Si}=\mathrm{CH}_{2},{ }^{24,25}$ and both only $0.014-0.012 \AA$ longer than that of the SiCF. Several stable silicon-carbon doubly bonded molecules have also been reported. ${ }^{22}$ These invariably have large bulky substituents to tame their inherent reactivity, with typical bond lengths ranging from $1.702-1.764 \AA$. To our knowledge, there are no known stable molecules with carbonsilicon triple bonds, but theory predicts a value of $1.604 \AA$ for linear $\mathrm{HC} \equiv \mathrm{SiH},{ }^{26}$ very close to the excited state value obtained for SiCF.

\section{B. Rotational and vibrational analysis}

The observed ${ }^{2} \Pi$ state spin-orbit coupling splitting in $\operatorname{SiCF}\left(-105.2 \mathrm{~cm}^{-1}\right)$ is somewhat larger than the -80 to -93 $\mathrm{cm}^{-1}$ values predicted in our ab initio study, similar to the

TABLE V. Experimental and ab initio spectroscopic parameters of SiCF.

\begin{tabular}{lccccc}
\hline \hline & \multicolumn{2}{c}{$\tilde{X}^{2} \Pi$} & & \multicolumn{2}{c}{$\tilde{A}^{2} \Sigma^{+}$} \\
\cline { 2 - 3 } \cline { 6 - 6 } Parameter & Experiment & Ab Initio & & Experiment & Ab Initio \\
\hline$T_{0} \mathrm{~cm}^{-1}$ & 0 & 0 & & $16518.804(1)$ & $17104^{\mathrm{a}}$ \\
$\omega_{1}\left(\mathrm{C}-\mathrm{F}\right.$ stretch) $\mathrm{cm}^{-1}$ & $1494.5(17)^{\mathrm{b}}$ & $1454^{\mathrm{c}}$ & & $1606.9^{\mathrm{d}}$ & $1595^{\mathrm{c}}$ \\
$\omega_{2}\left(\right.$ bend) $\mathrm{cm}^{-1}$ & $259.83(82)^{\mathrm{b}}$ & 250 & & 387.9 & 408 \\
$\omega_{3}\left(\mathrm{Si}-\mathrm{C}\right.$ stretch) cm $\mathrm{cm}^{-1}$ & $666.17(78)^{\mathrm{b}}$ & 640 & & 737.1 & 749 \\
$x_{23}$ & $5.57(121)^{\mathrm{b}}$ & $\ldots$ & & $\ldots$ & $\ldots$ \\
$\varepsilon$ & $-0.148(5)^{\mathrm{b}}$ & -0.199 & & $\ldots$ & $\ldots$ \\
Spin-orbit coupling constant, $A, \mathrm{~cm}^{-1}$ & $-103.68(112)^{\mathrm{b}}$ & -93 & & $\ldots$ & $\ldots$ \\
$\mathrm{C}-\mathrm{F}$ bond length $(\AA)$ & $\ldots$ & $1.288^{\mathrm{e}}$ & & $\ldots$ & $1.296^{\mathrm{e}}$ \\
SiC bond length $(\AA)$ & $1.692(1)$ & $1.703^{\mathrm{e}}$ & & $1.594(1)$ & $1.602^{\mathrm{e}}$ \\
\hline \hline
\end{tabular}

\footnotetext{
${ }^{\mathrm{a}} T_{0}$ calculated from CCSD(T)/aug-cc-pVTZ $T_{\mathrm{e}}$ and G96LYP/aug-cc-pVTZ frequencies, Ref. 7.

${ }^{\mathrm{b}}$ From our Renner-Teller analysis of the emission spectra. The numbers in parenthesis are $1 \sigma$ standard errors.

${ }^{\mathrm{c}}$ G96LYP/aug-cc-pVTZ values, Ref. 7.

${ }^{\mathrm{d}}$ Vibrational fundamental by subtracting Q-branch maxima from Table I.

${ }^{\mathrm{e}} \operatorname{CCSD}(\mathrm{T}) /$ aug-cc-pVTZ values, Ref. 7.
} 
underestimation in $\mathrm{SiCCl}\left(-115 \mathrm{~cm}^{-1}\right.$ theory vs $-159.4 \mathrm{~cm}^{-1}$ experiment). In the pure precession approximation, the predominant contribution to the excited state spin-rotation parameter $\gamma$ would come from the interaction with the ${ }^{2} \Pi_{i}$ ground state, given by the relation

$$
\gamma=2 A B l(l+1) /\left(E_{\Pi}-E_{\Sigma}\right),
$$

where $A$ is the spin-orbit coupling constant, $l$ is the angular momentum quantum number of the electron that gives rise to the $\Sigma$ and $\Pi$ states, and $B$ is the rotational constant of the $\Sigma$ state. The calculated value is $4.46 \times 10^{-3} \mathrm{~cm}^{-1}$ compared to $\gamma=1.9 \times 10^{-3} \mathrm{~cm}^{-1}$ determined experimentally, agreeing in sign but differing substantially in magnitude, as was found previously for $\mathrm{SiCH}{ }^{1}$

As summarized in Table V and Fig. 7, the ab initio predictions of the ground state vibrational and Renner-Teller parameters are in gratifyingly good agreement with experiment. As discussed previously, ${ }^{7}$ the stretching frequencies are anomalous, with $\omega_{3}$ (nominal $\mathrm{SiC}$ stretch $)=666 \mathrm{~cm}^{-1}$ much lower than the $1175 \mathrm{~cm}^{-1}$ and $1275 \mathrm{~cm}^{-1}$ values for $\mathrm{SiCH}$ and $\mathrm{SiCCl}$, respectively. This is because, in SiCF, the two stretching modes are not distinct, but are rather admixtures of the stretching internal coordinates and behave as the symmetric and antisymmetric stretching motions of a symmetric triatomic molecule. The bending frequency and Renner-Teller parameter are as expected from theory, and the predicted ground state energy level pattern including the Renner-Teller and spinorbit effects (see Fig. 6) was very useful in understanding the emission spectra.

As reproduced by our $a b$ initio calculations, substitution of more electronegative halogen substituents onto $\mathrm{SiCH}$ shifts the band origin of the ${ }^{2} \Sigma^{+}-{ }^{2} \Pi$ electronic transition substantially to the blue $\left(\mathrm{SiCH}=11766.7 \mathrm{~cm}^{-1}, \mathrm{SiCCl}\right.$ $\left.=15164 \mathrm{~cm}^{-1}, \mathrm{SiCF}=16518.8 \mathrm{~cm}^{-1}\right)$. Our CCSD $(\mathrm{T}) /$ aug-cc-pVTZ results ${ }^{7}$ overestimate the $T_{0}$ values by $300-800 \mathrm{~cm}^{-1}$, while the ground state spin-orbit constants are underestimated by $5 \%(\mathrm{SiCH})$ to $28 \%(\mathrm{SiCCl})$. As shown in Fig. 3, the $a b$ initio rotational constants are gratifyingly good at reproducing the low resolution contour of the SiCF $0-0$ band.

The derived excited state vibrational frequencies of SiCF are compared to our previous $a b$ initio values ${ }^{7}$ in Table V. The agreement is excellent and lends confidence to the assignments of the weak LIF bands. We note that the emission spectra of the $3_{0}^{1}$ and $2_{0}^{2}$ bands (Fig. 4) are very similar and involve primarily activity in $2 v_{2}^{\prime \prime}$, whereas we expected $3_{0}^{1}$ would emit more strongly down to $v_{3}^{\prime \prime}$. This initially cast some doubt on which LIF band was which, but the current assignments are the most consistent with the $a b$ initio frequencies. Since $3^{1}$ and $2^{2}$ are only $50 \mathrm{~cm}^{-1}$ apart, it is likely that the levels are in Fermi resonance, accounting for the similarity in their emission spectra.

\section{CONCLUSIONS}

After considerable effort, the SiCF free radical has been detected in the gas phase. Although $\mathrm{CF}_{3} \mathrm{SiF}_{3}$ did not produce $\mathrm{SiCF}$ in our electric discharge jet, $\mathrm{CF}_{3} \mathrm{Si}\left(\mathrm{CH}_{3}\right)_{3}$ and $\mathrm{CF}_{3} \mathrm{SiH}_{3}$ were found to be viable precursors that gave weak spectra that were positively attributable to the previously unknown $\mathrm{SiCF}$ species. High resolution rotationally resolved spectra of the $0-0$ band allowed a determination of the linear molecule silicon-carbon bond lengths as $\mathrm{r}^{\prime \prime}(\mathrm{Si}-\mathrm{C})=1.692(1) \AA$ and $\mathrm{r}^{\prime}(\mathrm{Si}-\mathrm{C})=1.594(1) \AA$ with $\mathrm{r}(\mathrm{C}-\mathrm{F})$ fixed at $a b$ initio values. Using $2 \mathrm{D}$-LIF and single vibronic level emission techniques, several LIF bands of SiCF were identified amidst a host of stronger signals from impurities produced in the discharge. In addition, the resolved emission spectra from the $0^{0}$ and $2^{1}$ levels were fitted to a Renner-Teller model, allowing the elucidation of the vibrational angular momentum coupling and spin-orbit effects in the ground state.

\section{ACKNOWLEDGMENTS}

T.C.S. thanks Professor T. Steimle for valuable advice and support in the implementation of the 2D-LIF and high resolution laser techniques. D.J.C. is very grateful to the staff of Ideal Vacuum Products LLC for making his sabbatical leave in Albuquerque in the spring of 2017 such an enjoyable and productive time. D.J.C. also thanks the students, faculty, and staff at the University of Kentucky for 34 years of stimulating opportunities in teaching and research. The authors thank Dr. Mohammed Gharaibeh of the Department of Chemistry, University of Jordan for efforts at the University of Kentucky to identify the weak SiCF LIF bands. This research was funded by Ideal Vacuum Products.

${ }^{1}$ T. C. Smith, H. Li, D. J. Clouthier, C. T. Kingston, and A. J. Merer, J. Chem. Phys. 112, 3662 (2000).

${ }^{2}$ T. C. Smith, H. Li, D. A. Hostutler, D. J. Clouthier, and A. J. Merer, J. Chem. Phys. 114, 725 (2001).

${ }^{3}$ T. C. Smith, H. Li, D. J. Clouthier, C. T. Kingston, and A. J. Merer, J. Chem. Phys. 112, 8417 (2000).

${ }^{4}$ S. He, H. Li, T. C. Smith, D. J. Clouthier, and A. J. Merer, J. Chem. Phys. 119, 10115 (2003).

${ }^{5}$ T. C. Smith, D. J. Clouthier, and T. C. Steimle, J. Chem. Phys. 115, 817 (2001).

${ }^{6}$ T. C. Smith, D. J. Clouthier, and T. C. Steimle, J. Chem. Phys. 115, 5047 (2001).

${ }^{7}$ C. J. Evans and D. J. Clouthier, J. Chem. Phys. 117, 6439 (2002).

${ }^{8}$ T. C. Smith, C. J. Evans, and D. J. Clouthier, J. Chem. Phys. 117, 6446 (2002).

${ }^{9}$ H. Harjanto, W. W. Harper, and D. J. Clouthier, J. Chem. Phys. 105, 10189 (1996).

${ }^{10}$ W. W. Harper and D. J. Clouthier, J. Chem. Phys. 106, 9461 (1997).

${ }^{11}$ D. L. Michalopoulos, M. E. Geusic, P. R. R. Langridge-Smith, and R. E. Smalley, J. Chem. Phys. 80, 3556 (1984).

${ }^{12} \mathrm{~S}$. Gerstenkorn and P. Luc, Atlas du Spectre D'Absorption de la Molecule d'Iode (Editions du CNRS, Paris, 1978); Rev. Phys. Appl. 14, 791 (1979).

${ }^{13}$ H. Knoeckel and E. Tiemann, Program IodineSpec5, http://www.iqo.unihannover.de.

${ }^{14}$ H. Beckers, H. Burger, P. Bursch, and I. Ruppert, J. Organomet. Chem. 316, 41 (1986).

${ }^{15}$ H. Beckers, H. Burger, and R. Eujen, J. Fluorine Chem. 27, 461 (1985).

${ }^{16}$ T. C. Smith, C. J. Evans, and D. J. Clouthier, J. Chem. Phys. 118, 1642 (2003).

${ }^{17} \mathrm{See}$ http://www.wiredchemist.com/chemistry/data/bond_energies_lengths. $\mathrm{html}$ for a table of typical bond lengths.

${ }^{18}$ H. Burger, R. Eujen, and P. Moritz, J. Organomet. Chem. 401, 249 (1991).

${ }^{19}$ See http://pgopher.chm.bris.ac.uk for PGOPHER, a Program for Simulating Rotational Structure, C. M. Western, University of Bristol.

${ }^{20} \mathrm{C}$. M. Western, "PGOPHER: A program for simulating rotational, vibrational and electronic spectra," J. Quant. Spectrosc. Radiat. Transfer 186, 221-242 (2016). 
${ }^{21}$ S. G. He and D. J. Clouthier, Comput. Phys. Commun. 178, 676 (2008).

${ }^{22}$ P. P. Power, Chem. Rev. 99, 3463 (1999).

${ }^{23}$ W. W. Harper, K. W. Waddell, and D. J. Clouthier, J. Chem. Phys. 107, 8829 (1997).
${ }^{24}$ S. Bailleux, M. Bogey, J. Breidung, H. Burger, R. Fajgar, Y. Liu, J. Pola, M. Senzlober, and W. Thiel, Angew. Chem., Int. Ed. Engl. 35, 2513 (1996).

${ }^{25}$ S. Bailleux, M. Bogey, J. Demaison, H. Burger, M. Senzlober, J. Breidung, W. Thiel, R. Fajgar, and J. Pola, J. Chem. Phys. 106, 10016 (1997).

${ }^{26}$ R. Stegmann and G. Frenking, J. Comput. Chem. 17, 781 (1996). 